\title{
Acute effects of MDMA (3,4-methylenedioxymethamphetamine) on EEG oscillations: alone and in combination with ethanol or THC (delta-9-tetrahydrocannabinol)
}

\author{
Marieke M. Lansbergen • Glenn J. H. Dumont • \\ Joop M. A. van Gerven • Jan K. Buitelaar • \\ Robbert-Jan Verkes
}

Received: 26 May 2010 / Accepted: 20 September 2010 / Published online: 6 October 2010

(C) The Author(s) 2010. This article is published with open access at Springerlink.com

\begin{abstract}
Rationale Typical users of 3,4-methylenedioxymethamphetamine (MDMA or "ecstasy") are polydrug users, combining MDMA with alcohol or cannabis [most active compound: delta-9-tetrahydrocannabinol (THC)].

Objectives The aim of the present study was to investigate whether co-administration of alcohol or THC with MDMA differentially affects ongoing electroencephalogram (EEG) oscillations compared to the administration of each drug alone.
\end{abstract}

\footnotetext{
M. M. Lansbergen $(\bowtie) \cdot$ G. J. H. Dumont • R.-J. Verkes Department of Psychiatry, Donders Institute for Brain, Cognition and Behaviour, Radboud University Nijmegen Medical Centre, Nijmegen, the Netherlands

e-mail: M.Lansbergen@psy.umen.nl
}

M. M. Lansbergen

e-mail: mariekelansbergen@hotmail.com

\section{G. J. H. Dumont}

Moleman Psychopharmacology,

Amerongen, the Netherlands

\section{J. K. Buitelaar}

Department of Cognitive Neuroscience, Donders Institute for Brain, Cognition and Behaviour, Radboud University Nijmegen Medical Centre,

Nijmegen, the Netherlands

\section{J. K. Buitelaar}

Karakter Child and Adolescent Psychiatry University Centre,

Nijmegen, the Netherlands

J. M. A. van Gerven

Centre for Human Drug Research (CHDR),

Leiden, the Netherlands
Methods In two separate experiments, 16 volunteers received four different drug conditions: (1) MDMA (100 mg); (2) alcohol clamp (blood alcohol concentration $=0.6 \%$ ) or THC (inhalation of 4, 6 and $6 \mathrm{mg}$, interval of 1.5 h); (3) MDMA in combination with alcohol or THC; and (4) placebo. Before and after drug administration, electroencephalography was recorded during an eyes closed resting state

Results Theta and alpha power increased after alcohol intake compared to placebo and reduced after MDMA intake. No interaction between alcohol and MDMA was found. Significant MDMA $\times$ THC effects for theta and lower-1-alpha power indicated that the power attenuation after the combined intake of MDMA and THC was less than the sum of each drug alone. For the lower-2-alpha band, the intake of MDMA or THC alone did not significantly affect power, but the intake of combined MDMA and THC significantly decreased lower-2-alpha power.

Conclusions The present findings indicate that the combined intake of MDMA and THC, but not of MDMA and alcohol, affects ongoing EEG oscillations differently than the sum of either one drug alone. Changes in ongoing EEG oscillations may be related to the impaired task performance that has often been reported after drug intake.

Keywords MDMA - Ecstasy - Alcohol - Ethanol - THC . Cannabis $\cdot$ Acute effects $\cdot$ Healthy volunteers $\cdot$ Neuronal oscillations $\cdot$ EEG

\section{Introduction}

Alcohol (ethanol), cannabis and ecstasy [3,4-methylenedioxymethamphetamine (MDMA)] are the most commonly 
used psychoactive substances for recreational purpose in Western society. Ecstasy users typically are polydrug users in that they combine the use of ecstasy frequently with that of alcohol or cannabis (Barrett et al. 2005; Boys et al. 2001; Gouzoulis-Mayfrank and Daumann 2006; Parrott 2007).

MDMA is a psychostimulant that produces euphoria, enhanced sociability and a sense of increased empathy (de la Torre et al. 2004). Although alcohol has both arousing and sedating effects that are dose-dependent with high inter-individual variability, at high blood alcohol concentrations $(\mathrm{BAC}>0.5 \mathrm{~g} / \mathrm{L}$ ), alcohol generally causes sedation (Gulick and Gould 2007). Cannabis produces relaxation, feelings of happiness and increased sensory perception, but it may also produce dizziness and feelings of depression (Green et al. 2003).

In the brain, MDMA increases the levels of available serotonin, dopamine and norepinephrine (Colado et al. 2004; Liechti and Vollenweider 2000, 2001; Mlinar and Corradetti 2003; Pifl et al. 1995; Sprague et al. 2004). MDMA is usually ingested orally and rapidly absorbed. Plasma levels peak at 1-2 $\mathrm{h}$ after drug intake, and maximum behavioural and subjective effects occur around the peak plasma levels (de la Torre et al. 2004; Dumont et al. 2009a).

Alcohol has direct and indirect effects on multiple neurotransmitter and neuropeptide systems (Little 1999; Vengeliene et al. 2008), such as the inhibition of glutamate and enhancement of inhibitory GABA neurotransmission. Oral administration of a single dose of alcohol results in a highly variable increase of BAC with maximal plasma concentrations occurring 30-60 min after ingestion, after which BAC decline steadily at an average rate of roughly $0.15 \mathrm{gL}^{-1} \mathrm{~h}^{-1}$.

The major active compound of cannabis is delta-9tetrahydrocannabinol (THC; Ilan et al. 2005). THC is an agonist for cannabinoid receptors (particularly CB1). After inhalation, THC is rapidly distributed from the blood into the brain and other lipophilic tissues, for which it has a high affinity. Consequently, peak plasma levels are reached within several minutes and show a rapid decline reflecting redistribution, whereas subjective and cognitive effects of cannabis inhalation peak around 15-60 min and last for several hours (Dumont et al. 2010c; Strougo et al. 2008).

Whereas the central effects of a high dose of alcohol are generally sedative, and consequently lead to non-specific cognitive impairments, THC and MDMA affect cognition more specifically. The cognitive effects of THC generally include, but are not restricted to, impairment of memory and psychomotor function and subjective relaxation and sedation (Zuurman et al. 2009). MDMA stimulates psychomotor speed but not accuracy and impairs memory function (Kuypers and Ramaekers 2007; Lamers et al. 2003). Although many reports addressed the acute cognitive effects of MDMA, a review by our group concluded that effects reported were generally small and inconsistent across studies (Dumont and Verkes 2006).

Since ecstasy (MDMA) is commonly used in combination with cannabis (THC) or alcohol (ethanol), it is important to examine the effects of the co-administration of MDMA and ethanol or MDMA and THC on subjective feelings and cognitive and psychomotor performance. In two separate studies, Dumont et al. (2008, 2010c) addressed this issue. Although co-administration of MDMA with ethanol reversed subjective feelings of reduced alertness caused by alcohol, MDMA did not affect ethanol-induced delays of psychomotor speed (Dumont et al. 2008). Combined intake of MDMA and ethanol did not exacerbate the impairment in memory or psychomotor performance (Dumont et al. 2008). Whereas MDMA stimulates psychomotor speed, co-administration of MDMA with THC could not significantly reverse the drowsiness or the impairments of psychomotor speed produced by THC (Dumont et al. 2010c). Similar to the combined intake of MDMA and ethanol, co-administration of MDMA with THC did not appear to exacerbate the impairments of memory (Dumont et al. 2010c).

The current paper will address the effects of these drug combinations on electroencephalogram (EEG), a parameter of brain function. EEG can be used to get more insight into ongoing EEG oscillations, which are a manifestation of neuronal oscillations. EEG oscillations may be related to a variety of factors such as levels of alertness (Oken et al. 2006) and information processing (e.g., Klimesch 1999) and are affected by drug intake. For example, drowsiness during an eyes closed resting condition is associated with increased theta power and decreased alpha and beta power (Oken et al. 2006). Furthermore, a role of EEG oscillations in human information processing such as attention, active inhibition, memory and integrative functions has been demonstrated (Klimesch 1999; Knyazev 2007; Sauseng et al. 2010). For example, a higher memory load in a working memory task has been related to increased theta and alpha power. Based on these and other findings, it has been suggested that theta oscillations play a role in memory functions and alpha oscillations in the active inhibition of visual processing (Jensen et al. 2002; Jensen and Tesche 2002). Psychoactive substances also affect EEG oscillations (Mitchell et al. 2008).

Only few studies examined the effects of the administration of MDMA, THC and ethanol on electrocortical activity during a resting state (i.e. ongoing EEG oscillations), and the effects of the co-administration of MDMA with either THC or ethanol have not been addressed so far. To our knowledge, only one published study investigated the acute effects of MDMA on EEG power during a resting condition in MDMAnaive volunteers, revealing decreased theta and alpha power after MDMA administration (Frei et al. 2001). 
Regarding THC effects on EEG power, the most consistent finding after acute cannabis administration is decreased theta power (Böcker et al. 2010; Ilan et al. 2004, 2005; Zuurman et al. 2008, 2010). THC effects on alpha power are more variable, varying from increased alpha power (but only during a state in which THC induced feelings of euphoria; Lukas et al. 1995) or no effects on alpha power (Böcker et al. 2010; Ilan et al. 2004, 2005; Zuurman et al. 2008, 2010) during a resting state. The decrease in theta power after cannabis use was dose-related and correlated with decreased working memory performance (Böcker et al. 2010). It has been speculated that decreased theta power after cannabis administration reflects the desynchronization of ongoing theta oscillations, which interferes with phase resetting of theta that is needed for optimal memory performance (Böcker et al. 2010). Another explanation that has been proposed is that theta power attenuation after cannabis intake may reflect increased activity of the default mode network (DMN), which interferes with information processing (Böcker et al. 2010). The DMN is a group of brain areas that are functionally related, active during rest and deactivated during externally directed behaviour (Greicius et al. 2003). It is hypothesized that activity in the DMN reflects mind wandering or internally oriented attention. However, the relation between theta oscillations and activity in the DMN has not been fully established, and activity in the DMN has also been related to alpha and beta oscillations and alpha synchronization (Jann et al. 2009; Laufs 2008).

The effects of acute ethanol intake on EEG power have been investigated more intensively (Little 1999). The acute administration of ethanol consistently increases theta and alpha power (particularly within the lower-1 alpha frequency range, 8-10 Hz; e.g. Ilan and Gevins 2001; Lukas et al. 1986; Stenberg et al. 1994; Tran et al. 2004), although alpha power returned to normal levels $60 \mathrm{~min}$ after ethanol administration (Lukas et al. 1986). Increased alpha power after alcohol consumption has been shown to correlate positively with feelings of euphoria (Lukas et al. 1986). Furthermore, a reduction in peak power frequency for theta and lower-2 alpha (Ehlers et al. 1989) has been demonstrated with alcohol consumption.

In summary, whereas the administration of MDMA alone or THC alone decreases theta power, intake of ethanol alone increases theta power. Similar patterns have been found for alpha power, which decreases after MDMA and increases after ethanol. The question remains whether combined intake of MDMA and THC or ethanol has an additive effect or an interaction effect on theta and alpha oscillations. In two separate prospective placebo-controlled within-subject studies, we aim to assess the effects of acute administration of MDMA on electrocortical power, alone and in combination with ethanol (study 1) or THC (study 2).
Based on previous EEG studies, we expect that MDMA decreases theta and alpha power, THC decreases theta but not alpha power, and ethanol increases theta and alpha power. The question remains whether ethanol could counteract the MDMA effects on theta and alpha power and whether MDMA and THC would exacerbate the theta power attenuation after a single drug intake.

\section{Methods}

Study design

Data were collected in two separate studies that have already been described in detail in recently published papers: (a) study 1: MDMA $\times$ ethanol interaction study (Dumont et al. 2008) and (b) study 2: MDMA $\times$ THC interaction study (Dumont et al. 2010c). Studies were similar in design; relevant discrepancies between studies are described in the pertaining sections and presented in Table 1. Both studies utilized a four-way, double-blind, randomized, crossover and placebo-controlled design. Sixteen volunteers were randomly assigned to one of four treatment sequences.

Study 1 Each volunteer received a capsule containing either $100 \mathrm{mg}$ MDMA or placebo and a placebo-controlled intravenous ethanol clamp as described below, with a washout of 7 days between treatments. So, each participant attended four drug sessions: (1) placebo+placebo, (2) MDMA+placebo (i.e. MDMA alone), (3) ethanol+placebo (i.e. ethanol alone) and (4) MDMA+ethanol.

Study 2 Each volunteer received a capsule containing either $100 \mathrm{mg}$ MDMA or placebo and inhaled THC or placebo vapour as described below, with a washout of 7 days between each treatment. So, each participant attended four drug sessions: (1) placebo+placebo, (2) MDMA+placebo (i.e. MDMA alone), (3) THC+placebo (i.e. THC alone) and (4) MDMA+THC.

\section{Participants}

Study 1 Participants included 16 healthy volunteers (nine men, seven women) who are regular users of ecstasy (at least eight exposures in the last 2 years) and alcohol (at least one exposure per week), $22.1 \pm 2.9$ (mean \pm SD)years of age (range 18-29). Lifetime ecstasy exposure was on average $95(\mathrm{SD}=138$, range 14-431). More detailed demographic data have been reported elsewhere (Dumont et al. 2008). 
Table 1 Time schedule of each day in the MDMA $\times$ ethanol and MDMA $\times$ THC study

\begin{tabular}{lll}
\hline Time & Study 1: MDMA $\times$ ethanol & Study 2: MDMA $\times$ THC \\
\hline 0845 & Light breakfast & Light breakfast \\
1000 & EEG recordings & EEG recordings \\
1030 & Intake of MDMA or placebo capsule & Intake of MDMA or placebo capsule and \\
& & 1st THC or placebo inhalation \\
1100 & Start ethanol or placebo infusion & 2nd THC or placebo inhalation \\
1200 & & EEG recordings \\
1230 & EEG recordings & Lunch \\
1400 & Lunch/stop ethanol or placebo infusion & \\
\hline
\end{tabular}

Study 2 Participants included 16 healthy volunteers (12 men, 4 women) who are regular users of ecstasy (at least eight exposures in the last 2 years) and THC (on average at least two exposures per week in the last year), aged 18-27. Detailed demographic data have been published elsewhere (Dumont et al. 2010c).

Participants for both studies were recruited through advertisements on the Internet and at local drug testing services. Exclusion criteria for both studies included pregnancy and (history of) psychiatric illness (assessed using the Structured Clinical Interview for DSM-IV Axis I disorders, nonpatient version; First et al. 1994). Axis II disorders were excluded using the Temperament and Character Inventory (Svrakic et al. 1993), use of over-the-counter medication within 2 months prior to the study start, (history of) treatment for addiction problems, excessive smoking $(>10$ cigarettes/day) and orthostatic hypotension. Physical and mental health was determined by assessment of medical history, a physical and ECG examination as well as by standard haematological and chemical blood examinations, all conducted by a medical practitioner.

The time between the two studies exceeded 6 months. Four volunteers participated in both studies. These volunteers were included in the studies according to the protocol and all study procedures were trained before each study started. The studies have been approved by the local Medical Ethics Committee and performed in accordance with the ethical standards laid down in the 1964 Declaration of Helsinki. All participants gave their written informed consent before participating in the study and were paid for their participation.

\section{Adverse events/missing data}

Study 1 One participant had a mild adverse reaction (local vascular reaction) during the ethanol infusion, which led to premature interruption of the procedure, and one other participant did not refrain from drug use; both (one man, one woman) were excluded from further participation and results obtained were not included in the data analysis.
Study 2 One participant did not refrain from drug use, after which further study participation was denied. Two participants experienced an adverse event that was judged to be likely related to study drug administration (one participant developed a short-lasting (55 s) heart rate increase of $>180 \mathrm{bpm}$ and another participant had mild hallucinations, the latter subsiding along with other drug effects). These participants were excluded from further participation; data of completed study days obtained prior to these adverse events were analysed as described.

\section{Study procedure}

Table 1 presents the time schedules of the events on a test day for each study that are relevant for the present paper. For a detailed description of a test day, see Dumont et al. $(2008,2010 \mathrm{c})$. In both studies, participants arrived in the morning and were offered a light breakfast after a negative urine drug screen (opiates, cocaine, benzodiazepines, amphetamines, methamphetamines and delta-9tetrahydrocannabinol) as well as a negative alcohol breath test and recording of signs and symptoms of possible health problems. EEG data were acquired at 1000 hours, $30 \mathrm{~min}$ prior to the first drug administration. Drug administration (MDMA or placebo capsule in study 1; MDMA or placebo capsule and THC or placebo inhalation in study 2) was scheduled at 1030 hours. The ethanol or placebo infusion in study 1 was started at 1100 hours for a duration of $3 \mathrm{~h}$. The second THC or placebo inhalation in study 2 was administered at 1200 hours. In both studies, postassessment of EEG was conducted at 1230 hours, at the time around the peak concentration of MDMA $(2 \mathrm{~h})$. Since subjective and cognitive effects of THC have been shown within 15-60 min after administration, the second THC inhalation was administered 30 min prior to the EEG data acquisition. Volunteers were offered lunch at 1400 hours and were sent home at 1700 hours after a medical check.

In addition to the EEG data, subjective, neuropsychological and physiological data were acquired, which have 
already partially been published (Dumont et al. 2008, 2009a, b, 2010a, b, c).

Drugs and dosages

\section{3,4-Methylenedioxymethamphetamine}

MDMA (or matched placebo) was given orally as a capsule in a single dose of $100 \mathrm{mg}$. MDMA was obtained from Lipomed AG, Arlesheim, Switzerland, and encapsulated according to Good Manufacturing Practice (GMP) by the Department of Clinical Pharmacy of the Radboud University Nijmegen Medical Centre. MDMA (100 mg) orally is a relevant dose in the range of normal single recreational dosages. Previous experiments in humans used doses up to $150 \mathrm{mg}$ without serious adverse events.

\section{Ethanol clamping}

To standardize alcohol kinetics and dynamics, an intravenous clamping procedure has been developed, which minimizes variations in BAC that are high after oral administration (Zoethout et al. 2008). Ethanol (or glucose $5 \%$ as its placebo) was administered continuously by intravenous infusion of $10 \%$ ethanol in $5 \%$ glucose solution, aimed to maintain an ethanol blood concentration of $0.6 \%$ o $(0.6 \mathrm{~g} / \mathrm{L})$ for $3 \mathrm{~h}$. The alcohol clamp was targeted at $0.6 \%$, by which we aimed to provide the equivalent of approximately two to three alcoholic beverages. This concentration is just above the legal limit for traffic participation in many Western countries and commonly used in social settings as it is considered to be a safe and relatively moderate level despite significant CNS effects (Zoethout et al. 2009). The infusion rate was calculated using frequent breath alcohol concentration measurements, according to a previously designed algorithm (Zoethout et al. 2008). Breath alcohol concentration was assessed using a HONAC AlcoSensor IV ${ }^{\circledR}$ Intoximeter. The process was semi-automated using a computer spreadsheet programme which uses changes in the measured breath alcohol concentrations to calculate the infusion rate that is needed to maintain the ethanol level at $0.6 \%$. The operator of the breath alcoholmeter and the ethanol infusion pump was unblinded for alcohol treatment, but did not communicate with the study team or the participant about the results at any stage during the trial and was not involved in the analyses. A sham procedure including a mock spreadsheet was used on ethanol-placebo occasions.

\section{delta-9-Tetrahydrocannabinol}

THC kinetics are highly variable in a free smoking procedure. To minimize variation due to inhalation volume and duration as well as THC content, a standardized procedure using a vaporizer, which has been shown to produce reliable and reproducible THC levels (Strougo et al. 2008), was used to administrate THC.

THC was purified according to GMP-compliant procedures (Farmalyse BV, Zaandam, the Netherlands) from the flowers of Cannabis sativa grown under Good Agricultural Practice (Bedrocan BV Medicinal Cannabis, Veendam, the Netherlands; Choi et al. 2004; Hazekamp et al. 2004). Each dose $(4,6$ and $6 \mathrm{mg})$ of THC ( $>98 \%$ purity by HPLC/ GC) was dissolved in $200 \mu \mathrm{L} 100$ vol.\% ethanol. THC was stored in the dark at $-20^{\circ} \mathrm{C}$ in $1-\mathrm{mL}$ amber glass vials containing a Teflon screw-cap secured with Parafilm to minimize evaporation. The solvent was used as placebo.

On each study day, THC (4, 6 and $6 \mathrm{mg})$ or placebo was administered at 90 -min intervals by inhalation using a Volcano ${ }^{\circledR}$ vaporizer (Storz-Bickel $\mathrm{GmbH}$, Tüttlingen, Germany), a validated method of intrapulmonary THC administration (Abrams et al. 2007; Hazekamp et al. 2006; Zuurman et al. 2008). The same regimen was also used with MDMA administration where $4 \mathrm{mg}$ THC was administered together with MDMA and the two subsequent doses of $6 \mathrm{mg}$ of THC were administered 90 and $180 \mathrm{~min}$ after MDMA administration. Within 5 min before administration, THC was vaporized at a temperature of about $225^{\circ} \mathrm{C}$ and the vapour was stored in a polythene bag equipped with a valved mouthpiece, preventing the loss of THC between inhalations. The transparant bag was covered with a black plastic bag to prevent unblinding. The personnel responsible for drug preparation was not involved in any other part of the study. Participants were not allowed to speak, were instructed to inhale deeply and hold their breath for $10 \mathrm{~s}$ after each inhalation. Within 2-3 $\mathrm{min}$, the bag was to be fully emptied. The inhalation procedure was practiced at screening using the solvent only. The inhalation schedule was predicted to cause THC plasma concentrations and effects corresponding to the THC contents in roughly one marijuana cigarette. The decision to proceed to the next higher THC dose was made by a physician based on adverse events and physical signs.

\section{Electrophysiological recordings}

The EEG data were recorded using an elastic cap with 27 tin electrodes according to the 10-20 electrode international system. Data were referenced to the left mastoid with the ground electrode placed at the right mastoid. Vertical electrooculogram was recorded from electrodes attached above and below the left eye and the horizontal electrooculogram from the outer canthi of both eyes. Electrode impedance was below $5 \mathrm{k} \Omega$ at the start of the recording session. EEG and EOG were amplified with a bandwidth of 
$0.005-120 \mathrm{~Hz}$. The sampling rate was $512 \mathrm{~Hz}$. Participants were asked to sit quietly for 3 min with eyes closed, during which resting-state EEG was recorded.

\section{Electrophysiological analysis}

EEG and EOG data were analyzed using the Brain Vision Analyzer software (www.brainproducts.com). EEG signals were re-referenced off-line to the average of all EEG electrodes, and the sampling rate was changed to $256 \mathrm{~Hz}$ with a high-pass filter of $0.04 \mathrm{~Hz}$, low-pass filter of $40 \mathrm{~Hz}$ and a notch filter of $50 \mathrm{~Hz}$. The 3-min continuous EEG data were segmented into 2-s epochs. First, a full segment baseline correction was applied. Subsequently, trials with artefacts were rejected from further analysis (absolute amplitude criterion of $120 \mu \mathrm{V}$ and low activity criterion of $0.5 \mu \mathrm{V}$ within a 100 -ms time window for EEG data). Then, ocular artefact correction was conducted according to the Gratton et al. (1983) algorithm. Finally, EEG data were Fourier-transformed (Hanning window length of 20\%) and subsequently lntransformed. Based on previous literature (e.g. Böcker et al. 2010; Ilan et al. 2004, 2005), we selected frontal (Fz, F3, F4), central (Cz, C3, C4) and parietal (Pz, P3, P4) electrodes for the analysis.

Since alpha oscillations show large interindividual differences (Klimesch 1999) and previous drug studies reported not only a change in absolute power of specific frequency bands but also in the peak frequency of alpha oscillations (Ehlers et al. 1989), the analysis of fixed frequency bands has major limitations. Klimesch (1999) suggested adjusting the frequency windows of alpha and theta for each individual by using individual alpha peak frequency (IAF) as an anchor point. More specifically, individual frequency bandwidths for alpha and theta bands are determined as a percentage of IAF (Doppelmayr et al. 1998). Moreover, Klimesch (1999) suggested dividing alpha oscillations into three frequency bands: lower- 1 alpha (i.e. $0.6 *$ IAF- $0.8 * \mathrm{IAF})$, lower- 2 alpha $(0.8 * \mathrm{IAF}-1 * \mathrm{IAF})$ and upper alpha (i.e. IAF-1.2*IAF). Similar to Klimesch (1999), in the present study, the power of individualdefined frequency bands was calculated. Individual frequency bands were calculated based on the mean IAF of each participant. First, for each participant, IAF was defined as the frequency at which alpha power was maximum within $7.5-15 \mathrm{~Hz}$ over the occipital electrodes $(\mathrm{O} 1, \mathrm{Oz}, \mathrm{O} 2)$ in the eyes closed condition. Visual inspection was conducted for peak frequencies occurring at the boundaries of the search window. Subsequently, power estimates were derived from the average for the theta $(0.4 * \mathrm{IAF}-0.6 * \mathrm{IAF})$, lower-1 alpha $(0.6 * \mathrm{IAF}-0.8 * \mathrm{IAF})$, lower-2 alpha $(0.8 * \mathrm{IAF}-1 * \mathrm{IAF})$ and upper alpha $(1 * \mathrm{IAF}-$ 1.2*IAF) bands (Doppelmayr et al. 1998).
Statistical analysis

Since several participants did not complete the whole experiment as described in detail above, multivariate analysis of variance with repeated measures MANOVA would limit the sample size. Therefore, mixed model analyses of variance (SPSS 16.0) were conducted to examine the effects of drug administration alone and in combination on logarithmically transformed EEG data, separately for each study (study 1: MDMA in combination with ethanol; study 2: MDMA in combination with THC). First, separately for each drug condition, IAF and theta, lower-1 alpha, lower-2 alpha and upper alpha power before drug administration were subtracted from IAF and power in the specific frequency band after drug administration, respectively. The difference value of power was then entered in the mixed model analysis of variance, separately for IAF and theta, lower-1 alpha, lower-2 alpha and upper alpha power. Participant was used as subject variable. Fixed factors were MDMA (MDMA vs. placebo), THC or ethanol (THC or ethanol vs. placebo). For theta, lower-1 alpha, lower2 alpha and upper alpha power, the fixed factors - lead [frontal (Fz, F3, F4) vs. central (Cz, C3, C4) vs. parietal (Pz, P3, P4)] and laterality (left vs. right vs midline) - were added to the model. Power values after each drug administration and at each electrode site were treated as repeated measurements. If the interaction between MDMA $\times$ THC (or ethanol) was significant, post hoc analyses were conducted to investigate the effects of MDMA alone, THC (or ethanol) alone and combined MDMA+THC (or MDMA+ethanol) relative to placebo. The alpha level of significance was set at 0.05 twotailed. Results are presented as mean \pm SD unless indicated otherwise.

Repeated measures ANOVA was used to examine the pharmacokinetics of the drugs (see also Dumont et al. 2009a, 2010b).

\section{Results}

Pharmacokinetics

Study 1 Mean MDMA maximal plasma concentration was $202.5 \pm 74.1 \mu \mathrm{g} / \mathrm{L} 150 \mathrm{~min}$ after drug intake, and its kinetics did not differ between single MDMA or combined MDMA and ethanol administration (see Dumont et al. 2010b for more details). Mean BAC during the pseudosteady state phase was $0.56 \pm 0.06 \%$.

Study 2 Mean MDMA maximal plasma concentration was $213.3 \pm 29.6 \mu \mathrm{g} / \mathrm{L}$ at $105 \mathrm{~min}$ after drug administration and showed minimal decline during the sampling period (on average $168.3 \pm 20.2 \mu \mathrm{g} / \mathrm{L}$ at $300 \mathrm{~min}$ after drug adminis- 
tration). MDMA kinetics did not differ between MDMA alone and MDMA plus THC drug conditions (see Dumont et al. 2009a for more details). THC and 11-OH-THC (one of its two most important metabolites) consistently showed peak concentrations $5 \mathrm{~min}$ after administration and declined thereafter, whereas 11-nor-9-carboxy-THC (the other important metabolite of THC) concentrations rose throughout the sampling period. Plasma THC and metabolite concentrations did not differ significantly between the THC-alone and MDMA+THC conditions (Dumont et al. 2009a).

Individual alpha peak frequency

\section{Study 1 (MDMA-ethanol interaction)}

All participants presented a clear peak in the alpha frequency band in all conditions, except for the ethanol alone condition of one participant and in three drug conditions (placebo, ethanol alone and MDMA+ethanol condition) of a second participant. For these data points, IAF and individual frequency bands could not be calculated. Therefore, these data points were excluded from the mixed model analyses of variance.

Table 2 presents mean IAF prior to and after drug administration, separately for the four conditions. IAF decreased significantly after ethanol administration and increased significantly after MDMA intake compared to placebo intake $\left[F_{(1,34.0)}=12.51, p=0.001\right.$ and $F_{(1,33.4)}=$ $19.17, p<0.001$, respectively]. No significant interaction effect was found between MDMA and ethanol.

\section{Study 2 (MDMA-THC interaction)}

All participants presented a clear peak in the alpha frequency band in all conditions, except for three participants in the placebo condition, two participants in the THC alone condition, two participants in the combined MDMA and
THC condition, and one participant in the MDMA alone condition. These data points were excluded from the mixed model analysis of variance.

Table 3 presents mean IAF prior to and after drug administration, separately for the four conditions. As expected from study 1, MDMA significantly increased $\mathrm{IAF}$ as compared to placebo $\left[F_{(1,37.8)}=15.15, p<0.001\right]$. No significant main effect of THC administration or a significant interaction effect of THC and MDMA was found.

Individual frequency bands

Since mean IAF was affected by the administration of ethanol and MDMA, the analysis of individual frequency bands is more valid than the analyses of fixed frequency bands. Based on the IAFs, mean power for theta, lower-1 alpha, lower-2 alpha and upper alpha were calculated (see "Methods" section).

Tables 2 and 3 present mean power of theta, lower-1 alpha, lower-2 alpha and upper alpha oscillations before and after drug administration for the four conditions from study 1 and study 2 , respectively. The mean width of each individual frequency bands is also reported. Note that the frequency bands of theta and lower-1 alpha coincide with the traditional fixed theta frequency band $(4-8 \mathrm{~Hz})$.

\section{Study 1}

The results of the mixed model analysis for mean 'individualized' theta power (mean range, 4.4-6.4 Hz) revealed a significant reduction of theta power after MDMA and an increased theta power after ethanol administration compared to placebo $\left[F_{(1,49.2)}=107.36, p<\right.$ $\left.0.001 ; F_{(1,38.8)}=22.63, p<0.001\right]$, without an interaction effect of MDMA and ethanol. Furthermore, a significant ethanol $\times$ lead interaction effect $\left[F_{(2,96.0)}=3.90, p=0.023\right]$ and MDMA $\times$ lead effect $\left[F_{(2,100.4)}=3.55, p=0.032\right]$

Table 2 Mean individualized alpha peak frequency and mean power of theta, lower-1 alpha, lower-2 alpha and upper-alpha oscillations (across frontal, central and parietal sites) before and after drug administration for study 1 (MDMA $\times$ ethanol)

\begin{tabular}{|c|c|c|c|c|c|c|c|c|}
\hline & \multicolumn{2}{|l|}{ Placebo } & \multicolumn{2}{|l|}{ MDMA } & \multicolumn{2}{|l|}{ Ethanol } & \multicolumn{2}{|c|}{ MDMA+ethanol } \\
\hline & Pre & Post & Pre & Post & Pre & Post & Pre & Post \\
\hline IAF & $10.5(0.9)$ & $10.5(0.9)$ & $10.4(1.0)$ & $10.9(1.1)$ & $10.5(0.7)$ & $10.1(0.8)$ & $10.4(0.9)$ & $10.5(0.9)$ \\
\hline Theta $(4.4-6.4 \mathrm{~Hz})$ & $0.24(0.13)$ & $0.27(0.12)$ & $0.23(0.11)$ & $0.19(0.07)$ & $0.21(0.09)$ & $0.28(0.16)$ & $0.23(0.11)$ & $0.21(0.09)$ \\
\hline Lower-1 alpha $(6.4-8.4 \mathrm{~Hz})$ & $0.27(0.19)$ & $0.30(0.18)$ & $0.28(0.18)$ & $0.21(0.10)$ & $0.25(0.17)$ & $0.37(0.26)$ & $0.27(0.19)$ & $0.27(0.15)$ \\
\hline Lower-2 alpha (8.4-10.4 Hz) & $0.40(0.21)$ & $0.42(0.21)$ & $0.48(0.25)$ & $0.42(0.19)$ & $0.42(0.20)$ & $0.56(0.24)$ & $0.41(0.26)$ & $0.45(0.26)$ \\
\hline Upper alpha $10.4-12.4 \mathrm{~Hz}$ ) & $0.50(0.28)$ & $0.53(0.28)$ & $0.52(0.24)$ & $0.51(0.25)$ & $0.48(0.19)$ & $0.58(0.24)$ & $0.44(0.23)$ & $0.49(0.27)$ \\
\hline
\end{tabular}

Standard deviations are given in parentheses

$I A F$ individualized alpha peak frequency 
Table 3 Mean individualized alpha peak frequency and mean power of theta, lower-1 alpha, lower-2 alpha and upper-alpha oscillations (across frontal, central and parietal sites) before and after drug administration for study 2 (MDMA $\times$ THC)

\begin{tabular}{|c|c|c|c|c|c|c|c|c|}
\hline & \multicolumn{2}{|l|}{ Placebo } & \multicolumn{2}{|l|}{ MDMA } & \multicolumn{2}{|l|}{$\mathrm{THC}$} & \multicolumn{2}{|c|}{$\mathrm{MDMA}+\mathrm{THC}$} \\
\hline & Pre & Post & Pre & Post & Pre & Post & Pre & Post \\
\hline IAF & $10.8(1.4)$ & $10.7(1.2)$ & $10.3(1.0)$ & $10.8(1.2)$ & $10.0(1.2)$ & $9.9(1.0)$ & $10.2(0.9)$ & $10.6(1.0)$ \\
\hline Theta $(4.4-6.4 \mathrm{~Hz})$ & $0.19(0.07)$ & $0.23(0.09)$ & $0.21(0.10)$ & $0.17(0.07)$ & $0.20(0.13)$ & $0.17(0.07)$ & $0.21(0.06)$ & $0.17(0.07)$ \\
\hline Lower-1 alpha $(6.4-8.4 \mathrm{~Hz})$ & $0.19(0.10)$ & $0.23(0.11)$ & $0.22(0.12)$ & $0.17(0.10)$ & $0.20(0.12)$ & $0.18(0.08)$ & $0.21(0.08)$ & $0.16(0.08)$ \\
\hline Lower-2 alpha $(8.4-10.4 \mathrm{~Hz})$ & $0.32(0.23)$ & $0.29(0.22)$ & $0.35(0.23)$ & $0.30(0.28)$ & $0.32(0.23)$ & $0.32(0.22)$ & $0.34(0.19)$ & $0.25(0.17)$ \\
\hline Upper alpha (10.4-12.4 Hz) & $0.32(0.24)$ & $0.29(0.25)$ & $0.31(0.19)$ & $0.26(0.22)$ & $0.31(0.20)$ & $0.33(0.22)$ & $0.32(0.17)$ & $0.26(0.20)$ \\
\hline
\end{tabular}

Standard deviations are given in parentheses

$I A F$ individualized alpha peak frequency

revealed the greatest effect of ethanol and MDMA over parietal sites. No significant interaction effects were found with the factor laterality.

Regarding alpha power, analyses with individualized lower-1 alpha (mean range, 6.4-8.4 Hz), lower-2 alpha (8.4 $10.4 \mathrm{~Hz})$ and upper alpha $(10.4-12.4 \mathrm{~Hz})$ confirmed previous findings of significantly enhanced alpha power after ethanol administration compared to placebo $\left[F_{(1,118.2)}=72.87, p<\right.$ $0.001, F_{(1,85.6)}=86.21, p<0.001, F_{(1,378.3)}=34.13, p<0.001$ for lower-1 alpha, lower-2 alpha, and upper alpha, respectively] and significantly attenuated alpha power after MDMA intake compared to the placebo condition $\left[F_{(1,190.8)}=152.27, p<0.001, F_{(1,77.3)}=44.76, p<0.001\right.$, $F_{(1,206.6)}=10.55, p=0.001$ for lower-1 alpha, lower-2 alpha, and upper alpha, respectively]. MDMA did not significantly interact with ethanol. A significant ethanol $\times$ lead interaction effect for lower- 1 alpha power $\left[F_{(2,141.5)}=\right.$ $3.29, p=0.040]$ revealed the greatest enhancement of lower-1 alpha power after ethanol over parietal sites. No significant interaction effects were found with the factor laterality.

\section{Study 2}

Mixed model analyses for mean individualized theta power (mean range, 4.4-6.4 Hz) and mean individualized lower-1 alpha power (mean range, 6.4-8.4 Hz) revealed similar results: Whereas EEG power increased after placebo, EEG power decreased after the administration of MDMA alone, THC alone, and combined MDMA and THC [significant main effects of $\operatorname{MDMA}\left(F_{(1,107.8)}=23.18, p<0.001 ; F_{(1,54.1)}=\right.$ 53.6, $p<0.001$ for theta and lower-1 alpha power, respectively) and THC $\left(F_{(1,90.3)}=7.8, p=0.006 ; F_{(1,31.3)}=13.07, p=\right.$ 0.001 for theta and lower-1 alpha power, respectively)]. Additionally, as shown in Figs. 1 and 2, significant THC $\times$ MDMA interaction effects $\left[F_{(1,110.6)}=17.2, p<0.001\right.$; $F_{(1,37.3)}=17.8, p<0.001$ for theta and lower-1 alpha power, respectively] indicated that the combined effect produced by the administration of MDMA and THC on theta and lower-1 alpha power is not equal to the sum of their separate effects. As illustrated in Figs. 1 and 2, the attenuation of power when drugs were taken in combination appears to be considerably less than the sum of the drugs alone. Post hoc analyses to compare placebo with drug alone and combined drug intake revealed significant MDMA-alone effects $\left[F_{(1,11.4)}=20.97, p=0.001 ; F_{(1,11.1)}=16.7, p=0.002\right.$ for theta and lower-1 alpha power, respectively] and combined MDMA-THC effects $\left[F_{(1,11.9)}=10.46, p=\right.$ $0.007 ; F_{(1,12.4)}=10.61, p=0.007$ for theta and lower-1 alpha power, respectively] and trends for THC-alone drug effect $\left[F_{(1,15.2)}=4.84, p=0.044 ; F_{(1,13.2)}=3.60, p=0.080\right.$ for theta and lower-1 alpha power, respectively]. No significant interaction effects were found with the factors laterality or lead.

Mixed model analysis for lower-2 alpha power (mean range, $8.4-10.4 \mathrm{~Hz}$ ) yielded a main effect of MDMA $\left[F_{(1,77.1)}=24.2, p<0.001\right]$, interaction effect of THC $\times$ MDMA $\left[F_{(1,62.8)}=6.3, p=0.015\right]$ and a significant interaction effect of THC $\times$ MDMA $\times$ lead $\left[F_{(1,85.0)}=3.2, p=\right.$ 0.047]. Post hoc tests per lead to compare placebo with drug alone and combined drug intake revealed that at frontal leads, the intake of MDMA or THC alone did not significantly affect lower-2 alpha power, whereas the combination of MDMA and THC significantly decreased lower-2-alpha power $\left[F_{(1,10.0)}=10.28, p=0.009\right]$. As illus-

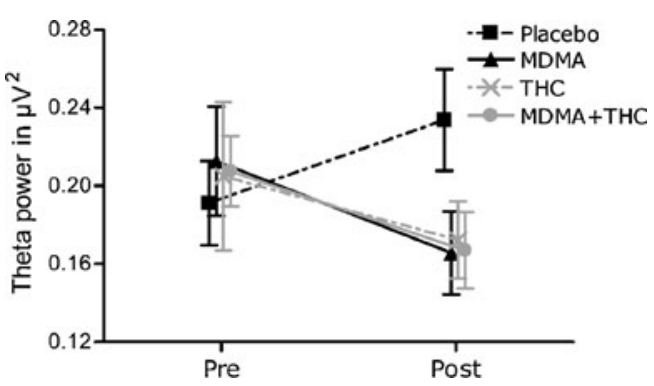

Fig. 1 Individualized theta power before (pre) and after (post) the administration of MDMA alone, THC alone, combined MDMA and THC, and placebo. Error bars represent the standard error of the mean 


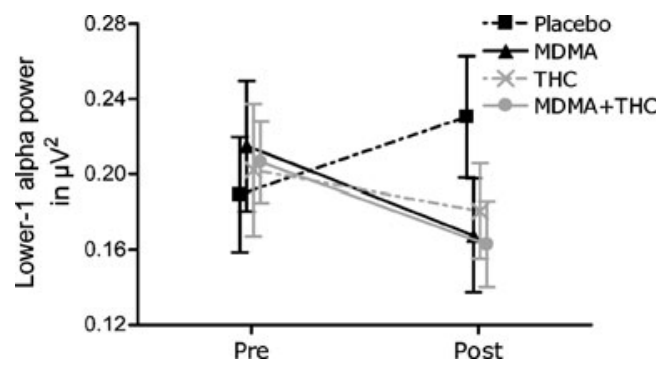

Fig. 2 Individualized lower-1 alpha power before (pre) and after (post) the administration of MDMA alone, THC alone, combined MDMA and THC, and placebo. Error bars represent the standard error of the mean

trated in Fig. 3, this pattern of drug effects was also present at parietal sites $\left[F_{(1,10.8)}=5.41, p=0.040\right]$. No significant interaction effects were found with the factor laterality.

Mixed model analysis for upper alpha power (mean range: $10.4-12.4 \mathrm{~Hz}$ ) revealed a significant main effect of MDMA $\left[F_{(1,185.3)}=27.9, p<0.001\right]$ and a MDMA $\times$ THC interaction effect $\left[F_{(1,68.6)}=6.09, p=0.016\right]$. Post hoc comparisons of each drug condition with the placebo condition did not yield any significant results, probably

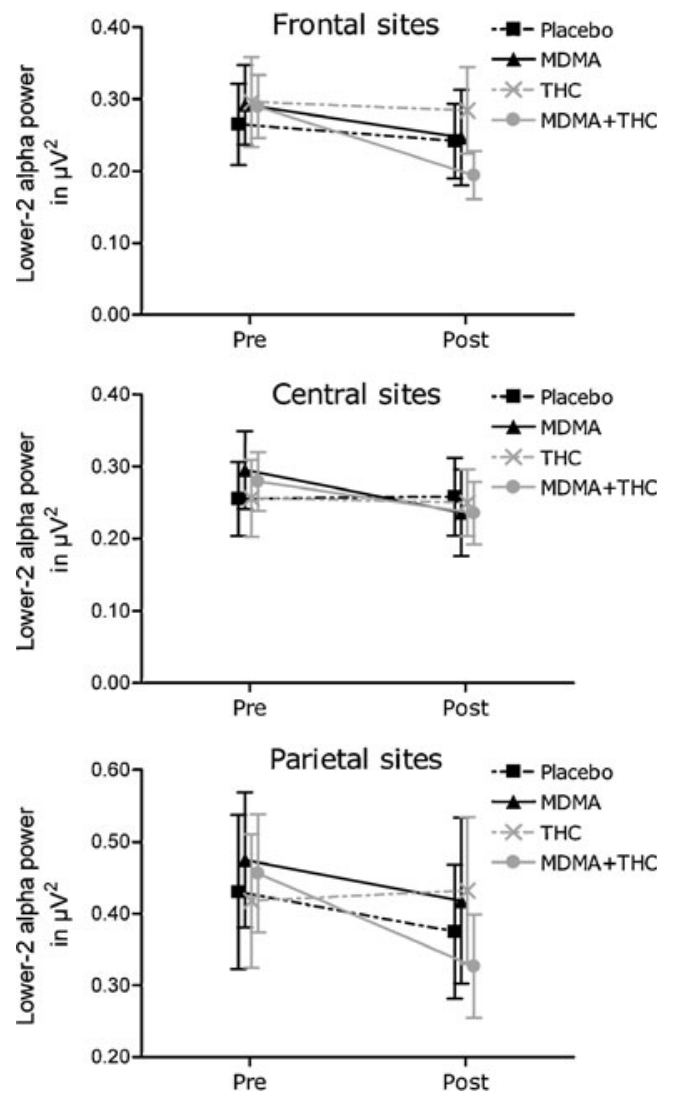

Fig. 3 Individualized lower-2 alpha power before (pre) and after (post) the administration of MDMA alone, THC alone, combined MDMA and THC, and placebo at frontal, central, and parietal sites. Error bars represent the standard error of the mean due to the large inter-individual variability (see standard deviations in Table 3). No significant interaction effects were found with the factors laterality or lead.

\section{Discussion}

The aim of the present report was to examine the effects of MDMA alone and in combination with (1) THC or (2) ethanol on ongoing EEG oscillations in healthy volunteers.

Consistent with previous findings (Ehlers et al. 1989), mean individual alpha peak frequency decreased after alcohol administration. In contrast, administration of MDMA increased individual alpha peak frequency. These findings indicate that it is very important to use individualized frequency bands to calculate EEG power (Klimesch 1999) instead of broad fixed frequency bands, which the majority of previous studies have used.

As expected from previous literature (Böcker et al. 2010; Frei et al. 2001; Little 1999), theta oscillations and lower-1 alpha oscillations, which overlap the traditional theta frequency band, were attenuated after the administration of MDMA alone and THC alone and increased after the intake of ethanol alone compared to placebo. Alpha oscillations were decreased after MDMA alone intake and increased after the administration of ethanol alone, also replicating previous findings (Frei et al. 2001; Little 1999). Consistent with the majority of previous studies (Böcker et al. 2010; Ilan et al. 2004, 2005; Zuurman et al. 2008, 2010), no significant changes for alpha power after THC administration were found. Note that increases in alpha power after THC intake have been reported, especially during THC-induced feelings of euphoria (Lukas et al. 1995), leading to the hypothesis that fluctuations in alpha power after THC administration coincide with fluctuations in feelings of euphoria (Lukas et al. 1995). Since EEG data and feelings of euphoria were not continuously recorded in the present study, this hypothesis could not be tested.

This is the first study that investigated the effects of the co-administration of THC or ethanol with MDMA on ongoing EEG oscillations. Ethanol and MDMA did not interact with regard to their effects on EEG power, i.e. the effects of combined intake was similar to the sum of the single drug effects. Regarding the co-administration of THC and MDMA, combined intake of MDMA and THC affected theta and lower-1 alpha power less than the sum of the single drug effects, but exacerbated the reductions in lower-2 alpha power. Although no corrections were made for multiple comparisons, drug effects appeared to be relatively large and main drug effects were consistent with previous literature.

What could be the functional interpretation of the present findings? Based on the suggestion that EEG oscillations are 
related to alertness (Oken et al. 2006), MDMA, THC and alcohol may generally affect alertness and consequently change theta and alpha power. However, single drug effects on alertness (i.e. decreased feelings of alertness by THC and alcohol, but not by MDMA; Dumont et al. 2008, 2010c) do not match the drug effects on EEG oscillations (i.e. decreased power by MDMA and THC and increased power by ethanol).

Recent animal and human studies investigating taskinduced EEG oscillations indicate that EEG oscillations are related to specific cognitive processes rather than general alertness. More specifically, it has been suggested that taskinduced increases in theta power and the phase relationship between theta and gamma oscillations are important for memory processes (Duzel et al. 2010; Kahana 2006; Klimesch 1999; Knyazev 2007). Recently, it has also been argued that theta oscillations reflect a "more general brain integrative mechanism" rather than an integrative mechanism specific for memory processes (Sauseng et al. 2010). Task-induced alpha oscillations have been related to topdown inhibitory control processes of visual information (Jensen et al. 2002; Knyazev 2007; Sauseng et al. 2009), necessary for optimal task performance. Ongoing background EEG oscillations may also be closely related to specific cognitive processes. For example, a significant association between decreased theta power at rest and impaired working memory performance after cannabis use has been reported (Böcker et al. 2010). The present finding that co-administration of MDMA with either THC or alcohol did not exacerbate single drug-induced effects on theta and lower-1 alpha power corresponds to the previous findings of Dumont et al. $(2008,2010 \mathrm{c})$ that the coadministration of MDMA with either THC or alcohol did not exacerbate single drug-induced impairments in memory and psychomotor performance. In contrast, the coadministration of MDMA with THC did exacerbate the reductions in lower-2 alpha power, which may be viewed as a contrasting finding at first glance. However, lower-2 alpha power has been specifically related to general attentional processes, whereas upper alpha power seems to be associated with semantic memory processes (Klimesch 1999). Therefore, the co-administration of MDMA with THC may not exacerbate the impairment in memory and psychomotor performance, but it may enhance attentional impairment.

How could changes in ongoing EEG oscillations be associated with cognitive impaired performance? It has been speculated that decreased theta power at rest after cannabis administration reflects desynchronization of ongoing theta oscillations, which interferes with phase resetting of theta that is needed for optimal memory performance (Böcker et al. 2010). Following this reasoning, one may predict that the increased theta power after alcohol intake leads to improved working memory performance. However, impaired memory has been reported after alcohol intake (Dumont et al. 2008). It may be speculated that the phase reset of oscillations and the phase relationship between different oscillations (i.e. cross-spectrum frequency coupling) are important for human information processing rather than the general increase/decrease in power. Subsequently, it could be speculated that both an increase and decrease of ongoing theta oscillations during a resting condition interfere with phase resetting of theta oscillations, necessary for correct behavioural performance. Therefore, decreased as well as increased ongoing theta oscillations may lead to incorrect phase reset of theta oscillations after a taskrelevant stimulus, resulting in reduced task-induced theta and impaired performance. Correspondingly, it has recently been demonstrated that ethanol reduces the normally observed taskinduced theta oscillations in healthy volunteers (Boha et al. 2009). The same mechanism may apply to alpha oscillations. Ongoing alpha oscillations in the present study were decreased after administration of MDMA alone and combined administration of THC and MDMA, but increased after ethanol intake. It may be speculated that decreased and increased ongoing alpha oscillations lead to incorrect phase reset of alpha oscillations during task performance, resulting in reduced task-induced alpha oscillations and impaired performance. Future studies should test this hypothesis and investigate how decreased as well as increased synchronization of ongoing EEG oscillations might hamper phase resetting.

Since MDMA, ethanol and THC influence multiple neurotransmitter systems (e.g. (Vengeliene et al. 2008), it is difficult to elucidate the mechanisms underlying the drug effects on ongoing EEG oscillations. The effects of ethanol have been suggested to be mediated by increasing the action of the inhibitory neurotransmitter GABA (Mitchell et al. 2008), whereas the modulation of EEG after MDMA is suggested to be predominantly mediated by the $5-\mathrm{HT}$ and NA system (Frei et al. 2001). The literature offers no clear explanation on whether or how cannabinoids affect EEG oscillations. Therefore, it is difficult to predict how the EEG would be affected by interactions of these neurotransmitter changes following different drug combinations. The results may suggest that the cannabinoid system has additive effects on the GABAergic system, where CB1 receptors are located on GABAergic neurons, but is infra-additive with respect to the blockade of presynaptic transporters, which might by and in itself create maximal reverse CB1 transmission, preventing additive effects of THC.

In conclusion, administration of MDMA, THC and ethanol change ongoing EEG oscillations, as reflected, in this study, in changes in theta and alpha power. Moreover, combined intake of MDMA and THC, but not of MDMA and alcohol, affects ongoing EEG oscillations differently than the sum of either one drug alone. Future studies may 
examine whether these changes are related to the impaired cognitive performance that has previously been found after acute drug administration.

Acknowledgements This research was supported by a grant from ZonMW (31000062), the Netherlands, and complies with current laws. We thank Jan Leytens for his assistance during the experiments. We also thank Rogier Donders for his advice on the statistical analyses.

Conflicts of interest Authors have no conflicts of interest.

Open Access This article is distributed under the terms of the Creative Commons Attribution Noncommercial License which permits any noncommercial use, distribution, and reproduction in any medium, provided the original author(s) and source are credited.

\section{References}

Abrams DI, Vizoso HP, Shade SB, Jay C, Kelly ME, Benowitz NL (2007) Vaporization as a smokeless cannabis delivery system: a pilot study. Clin Pharmacol Ther 82:572-578

Barrett SP, Gross SR, Garand I, Pihl RO (2005) Patterns of simultaneous polysubstance use in Canadian rave attendees. Subst Use Misuse 40:1525-1537

Böcker KBE, Hunault CC, Gerritsen J, Kruidenier M, Mensinga TT, Kenemans JL (2010) Cannabinoid modulations of resting state EEG theta power and working memory are correlated in humans. J Cogn Neurosci 22:1906-1916

Boha R, Molnar M, Gaal ZA, Czigler B, Rona K, Kass K et al (2009) The acute effect of low-dose alcohol on working memory during mental arithmetic: I. Behavioral measures and EEG theta band spectral characteristics. Int J Psychophysiol 73:133-137

Boys A, Marsden J, Strang J (2001) Understanding reasons for drug use amongst young people: a functional perspective. Health Educ Res 16:457-469

Choi YH, Hazekamp A, Peltenburg-Looman AM, Frederich M, Erkelens C, Lefeber AW et al (2004) NMR assignments of the major cannabinoids and cannabiflavonoids isolated from flowers of Cannabis sativa. Phytochem Anal 15:345-354

Colado MI, O'Shea E, Green AR (2004) Acute and long-term effects of MDMA on cerebral dopamine biochemistry and function. Psychopharmacology (Berl) 173:249-263

de la Torre R, Farre M, Roset PN, Pizarro N, Abanades S, Segura M et al (2004) Human pharmacology of MDMA: pharmacokinetics, metabolism, and disposition. Ther Drug Monit 26:137-144

Doppelmayr M, Klimesch W, Pachinger T, Ripper B (1998) Individual differences in brain dynamics: important implications for the calculation of event-related band power. Biol Cybern 79:49-57

Dumont GJ, Verkes RJ (2006) A review of acute effects of 3,4methylenedioxymethamphetamine in healthy volunteers. J Psychopharmacol 20:176-187

Dumont GJ, Wezenberg E, Valkenberg MM, de Jong CA, Buitelaar JK, van Gerven JM et al (2008) Acute neuropsychological effects of MDMA and ethanol (co-)administration in healthy volunteers. Psychopharmacology (Berl) 197:465-474

Dumont GJ, Kramers C, Sweep FC, Touw DJ, van Hasselt JG, de Kam $M$ et al (2009a) Cannabis coadministration potentiates the effects of "ecstasy" on heart rate and temperature in humans. Clin Pharmacol Ther 86:160-166

Dumont GJ, Sweep FC, van der Steen R, Hermsen R, Donders AR, Touw DJ et al (2009b) Increased oxytocin concentrations and prosocial feelings in humans after ecstasy (3,4-methylenedioxymethamphetamine) administration. Soc Neurosci 4:359-366

Dumont GJ, Kramers C, Sweep FC, Willemsen JJ, Touw DJ, Schoemaker RC et al (2010a) Ethanol co-administration moderates 3,4-methylenedioxymethamphetamine effects on human physiology. J Psychopharmacol 24:165-174

Dumont GJ, Schoemaker RC, Touw DJ, Sweep FC, Buitelaar JK, van Gerven JM et al (2010b) Acute psychomotor effects of MDMA and ethanol (co-)administration over time in healthy volunteers. $\mathrm{J}$ Psychopharmacol 24:155-164

Dumont GJ, van Hasselt JGC, de Kam M, van Gerven JM, Touw DJ, Buitelaar JK et al (2010c) Acute psychomotor, memory and subjective effects of MDMA and THC (co-)administration over time in healthy volunteers. J Psychopharmacol (in press). doi: $10.1177 / 0269881110376687$

Duzel E, Penny WD, Burgess N (2010) Brain oscillations and memory. Curr Opin Neurobiol 20:143-149

Ehlers CL, Wall TL, Schuckit MA (1989) EEG spectral characteristics following ethanol administration in young men. Electroencephalogr Clin Neurophysiol 73:179-187

First MB, Frances AJ, Pincus HA, Vettorello N, Davis WW (1994) DSMIV in progress. Changes in substance-related, schizophrenic, and other primarily adult disorders. Hosp Community Psychiatry 45:18-20

Frei E, Gamma A, Pascual-Marqui R, Lehmann D, Hell D, Vollenweider FX (2001) Localization of MDMA-induced brain activity in healthy volunteers using low resolution brain electromagnetic tomography (LORETA). Hum Brain Mapp 14:152-165

Gouzoulis-Mayfrank E, Daumann J (2006) The confounding problem of polydrug use in recreational ecstasy/MDMA users: a brief overview. J Psychopharmacol 20:188-193

Gratton G, Coles MG, Donchin E (1983) A new method for off-line removal of ocular artifact. Electroencephalogr Clin Neurophysiol $55: 468-484$

Green B, Kavanagh D, Young R (2003) Being stoned: a review of self-reported cannabis effects. Drug Alcohol Rev 22:453-460

Greicius MD, Krasnow B, Reiss AL, Menon V (2003) Functional connectivity in the resting brain: a network analysis of the default mode hypothesis. Proc Natl Acad Sci USA 100(1):253-258

Gulick D, Gould TJ (2007) Acute ethanol has biphasic effects on short- and long-term memory in both foreground and background contextual fear conditioning in C57BL/6 mice. Alcohol Clin Exp Res 31:1528-1537

Hazekamp A, Choi YH, Verpoorte R (2004) Quantitative analysis of cannabinoids from Cannabis sativa using ${ }^{1} \mathrm{H}-\mathrm{NMR}$. Chem Pharm Bull (Tokyo) 52:718-721

Hazekamp A, Ruhaak R, Zuurman L, van Gerven J, Verpoorte R (2006) Evaluation of a vaporizing device (Volcano) for the pulmonary administration of tetrahydrocannabinol. J Pharm Sci 95:1308-1317

Ilan AB, Gevins A (2001) Prolonged neurophysiological effects of cumulative wine drinking. Alcohol 25:137-152

Ilan AB, Smith ME, Gevins A (2004) Effects of marijuana on neurophysiological signals of working and episodic memory. Psychopharmacology (Berl) 176:214-222

Ilan AB, Gevins A, Coleman M, ElSohly MA, de Wit H (2005) Neurophysiological and subjective profile of marijuana with varying concentrations of cannabinoids. Behav Pharmacol 16:487-496

Jann K, Dierks T, Boesch C, Kottlow M, Strik W, Koenig T (2009) BOLD correlates of EEG alpha phase-locking and the fMRI default mode network. Neuroimage 45(3):903-916

Jensen O, Tesche CD (2002) Frontal theta activity in humans increases with memory load in a working memory task. Eur J Neurosci 15:1395-1399

Jensen O, Gelfand J, Kounios J, Lisman JE (2002) Oscillations in the alpha band $(9-12 \mathrm{~Hz})$ increase with memory load during retention in a short-term memory task. Cereb Cortex 12:877-882 
Kahana MJ (2006) The cognitive correlates of human brain oscillations. J Neurosci 26:1669-1672

Klimesch W (1999) EEG alpha and theta oscillations reflect cognitive and memory performance: a review and analysis. Brain Res Rev 29:169-195

Knyazev GG (2007) Motivation, emotion, and their inhibitory control mirrored in brain oscillations. Neurosci Biobehav Rev 31:377-395

Kuypers KP, Ramaekers JG (2007) Acute dose of MDMA (75 mg) impairs spatial memory for location but leaves contextual processing of visuospatial information unaffected. Psychopharmacology (Berl) 189(4):557-563

Lamers CT, Ramaekers JG, Muntjewerff ND, Sikkema KL, Samyn N, Read NL et al (2003) Dissociable effects of a single dose of ecstasy (MDMA) on psychomotor skills and attentional performance. J Psychopharmacol 17(4):379-387

Laufs H (2008) Endogenous brain oscillations and related networks detected by surface EEG-combined fMRI. Hum Brain Mapp 29 (7):762-769

Liechti ME, Vollenweider FX (2000) Acute psychological and physiological effects of MDMA ("ecstasy") after haloperidol pretreatment in healthy humans. Eur Neuropsychopharmacol 10:289-295

Liechti ME, Vollenweider FX (2001) Which neuroreceptors mediate the subjective effects of MDMA in humans? A summary of mechanistic studies. Hum Psychopharmacol 16:589-598

Little HJ (1999) The contribution of electrophysiology to knowledge of the acute and chronic effects of ethanol. Pharmacol Ther $84: 333-353$

Lukas SE, Mendelson JH, Benedikt RA, Jones B (1986) EEG alpha activity increases during transient episodes of ethanol-induced euphoria. Pharmacol Biochem Behav 25:889-895

Lukas SE, Mendelson JH, Benedikt R (1995) Electroencephalographic correlates of marihuana-induced euphoria. Drug Alcohol Depend 37:131-140

Mitchell DJ, McNaughton N, Flanagan D, Kirk IJ (2008) Frontalmidline theta from the perspective of hippocampal "theta". Prog Neurobiol 86:156-185

Mlinar B, Corradetti R (2003) Endogenous 5-HT, released by MDMA through serotonin transporter- and secretory vesicle-dependent mechanisms, reduces hippocampal excitatory synaptic transmission by preferential activation of 5-HT1B receptors located on CA1 pyramidal neurons. Eur J Neurosci 18:1559-1571

Oken BS, Salinsky MC, Elsas SM (2006) Vigilance, alertness, or sustained attention: physiological basis and measurement. Clin Neurophysiol 117:1885-1901

Parrott AC (2007) Ecstasy versus alcohol: Tolstoy and the variations of unhappiness. J Psychopharmacol 21:3-6

Pifl C, Drobny H, Reither H, Hornykiewicz O, Singer EA (1995) Mechanism of the dopamine-releasing actions of amphetamine and cocaine: plasmalemmal dopamine transporter versus vesicular monoamine transporter. Mol Pharmacol 47:368-373

Sauseng P, Klimesch W, Heise KF, Gruber WR, Holz E, Karim AA et al (2009) Brain oscillatory substrates of visual short-term memory capacity. Curr Biol 19:1846-1852

Sauseng P, Griesmayr B, Freunberger R, Klimesch W (2010) Control mechanisms in working memory: a possible function of EEG theta oscillations. Neurosci Biobehav Rev 34:1015-1022

Sprague JE, Brutcher RE, Mills EM, Caden D, Rusyniak DE (2004) Attenuation of 3, 4-methylenedioxymethamphetamine (MDMA, ecstasy)-induced rhabdomyolysis with alpha1-plus beta3adrenoreceptor antagonists. Br J Pharmacol 142:667-670

Stenberg G, Sano M, Rosen I, Ingvar DH (1994) EEG topography of acute ethanol effects in resting and activated normals. J Stud Alcohol 55:645-656

Strougo A, Zuurman L, Roy C, Pinquier JL, van Gerven JM, Cohen AF et al (2008) Modelling of the concentration-effect relationship of THC on central nervous system parameters and heart rate-insight into its mechanisms of action and a tool for clinical research and development of cannabinoids. J Psychopharmacol 22:717726

Svrakic DM, Whitehead C, Przybeck TR, Cloninger CR (1993) Differential diagnosis of personality disorders by the seven-factor model of temperament and character. Arch Gen Psychiatry 50:991-999

Tran Y, Craig A, Bartrop R, Nicholson G (2004) Time course and regional distribution of cortical changes during acute alcohol ingestion. Int J Neurosci 114:863-878

Vengeliene V, Bilbao A, Molander A, Spanagel R (2008) Neuropharmacology of alcohol addiction. Br J Pharmacol 154:299-315

Zoethout RW, van Gerven JM, Dumont GJ, Paltansing S, van Burgel $\mathrm{ND}$, van der Linden $\mathrm{M}$ et al (2008) A comparative study of two methods for attaining constant alcohol levels. $\mathrm{Br} \mathrm{J}$ Clin Pharmacol 66:674-681

Zoethout RW, Schoemaker RC, Zuurman L, van Pelt H, Dahan A, Cohen AF et al (2009) Central nervous system effects of alcohol at a pseudo-steady-state concentration using alcohol clamping in healthy volunteers. Br J Clin Pharmacol 68:524-534

Zuurman L, Roy C, Schoemaker RC, Hazekamp A, den Hartigh J, Bender JC et al (2008) Effect of intrapulmonary tetrahydrocannabinol administration in humans. J Psychopharmacol 22:707716

Zuurman L, Ippel AE, Moin E, van Gerven JM (2009) Biomarkers for the effects of cannabis and THC in healthy volunteers. Br J Clin Pharmacol 67:5-21

Zuurman L, Roy C, Schoemaker RC, Amatsaleh A, Guimaeres L, Pinquier JL et al (2010) Inhibition of THC-induced effects on the central nervous system and heart rate by a novel CB1 receptor antagonist AVE1625. J Psychopharmacol 24:363-371 